\title{
Master integrals for bipartite cuts of three-loop photon self energy
}

\author{
R.N. Lee ${ }^{a}$ and A.I. Onishchenko ${ }^{b, a, c}$ \\ ${ }^{a}$ Theory department, Budker Institute of Nuclear Physics, \\ Novosibirsk, Russia \\ ${ }^{b}$ Bogoliubov Laboratory of Theoretical Physics, Joint Institute for Nuclear Research, \\ Dubna, Russia \\ ${ }^{c}$ Skobeltsyn Institute of Nuclear Physics, Moscow State University, \\ Moscow, Russia \\ E-mail: r.n.lee@inp.nsk.su, onish@theor.jinr.ru
}

ABSTRACT: We calculate the master integrals for bipartite cuts of the three-loop propagator QED diagrams. These master integrals determine the spectral density of the photon self energy. Our results are expressed in terms of the iterated integrals, which, apart from the $4 m$ cut (the cut of 4 massive lines), reduce to Goncharov's polylogarithms. The master integrals for $4 m$ cut have been calculated in our previous paper in terms of the one-fold integrals of harmonic polylogarithms and complete elliptic integrals. We provide the threshold and high-energy asymptotics of the master integrals found, including those for $4 \mathrm{~m}$ cut.

KEYwORDS: NLO Computations

ArXiv EPrint: 2012.04230 


\section{Contents}

1 Introduction 1

2 Prototypes, LiteRed bases and boundary conditions 2

$\begin{array}{lll}3 & \text { Results } & 9\end{array}$

4 Asymptotics 10

5 Conclusion $\quad 12$

$\begin{array}{ll}\text { A Leading terms of } \epsilon \text {-expansion for LiteRed master integrals } & 13\end{array}$

\section{Introduction}

Photon self-energy operator $\Pi(s)=\sum_{n}\left(\frac{\alpha}{\pi}\right)^{n} \Pi_{n}(s)$ is a fundamental physical quantity of quantum electrodynamics. It is an important ingredient of many physically relevant calculations. One-loop result $\Pi_{1}(s)$ is presented in many QED textbooks (see, e.g., [1]), and the two-loop contribution $\Pi_{2}(s)$ has been calculated long ago by Källen and Sabry in ref. [2]. The three-loop contribution also appears in many applications, see, in particular, refs. [3-5]. Some calculations require knowing this object with very high precision. Kinoshita and Lindquist have derived 6-fold parametric representation for the renormalized three-loop photon self-energy in a dedicated work [6]. Except for this computationally quite expensive representation, no exact analytical representation for $\Pi_{3}(s)$ has been derived so far. Baikov and Broadhurst [3] have derived a simple Padè approximation for this operator using a few terms of the asymptotic expansions near 3 special points: $s=0,4 m^{2}, \infty$ ( $m$ is the electron mass). The precision of this approximation has been estimated indirectly, by comparing the contribution of the 3-loop polarization operator to 4-loop $g_{\mu}-2$. The conservative estimate of this precision given by Baikov and Broadhurst was as high as 0.002 percent for this case. This declared precision is quite remarkable and it would be interesting to compare the exact result with the approximate one in a more direct way. In particular, we are concerned about the impact of the second threshold $s=16 \mathrm{~m}^{2}$ which becomes relevant starting from three loops.

In the present paper we make an important step towards analytic calculation of the 3-loop polarization operator. Namely, we derive the analytic expressions for all master integrals which are required for the calculation of the spectral density $\rho(s)=\Im \Pi_{3}(s+i 0) / \pi$. Thanks to Cutkosky rules, this spectral density is expressed via bipartite cuts of a specific set of three-loop massive diagrams depicted in figure 1.

Classifying the cuts by the number of massive and massless lines that are cut, we have $(2,0),(2,1),(2,2)$, and $(4,0)$ cuts, where $(k, l)$ denotes the cut with $k$ massive and $l$ 

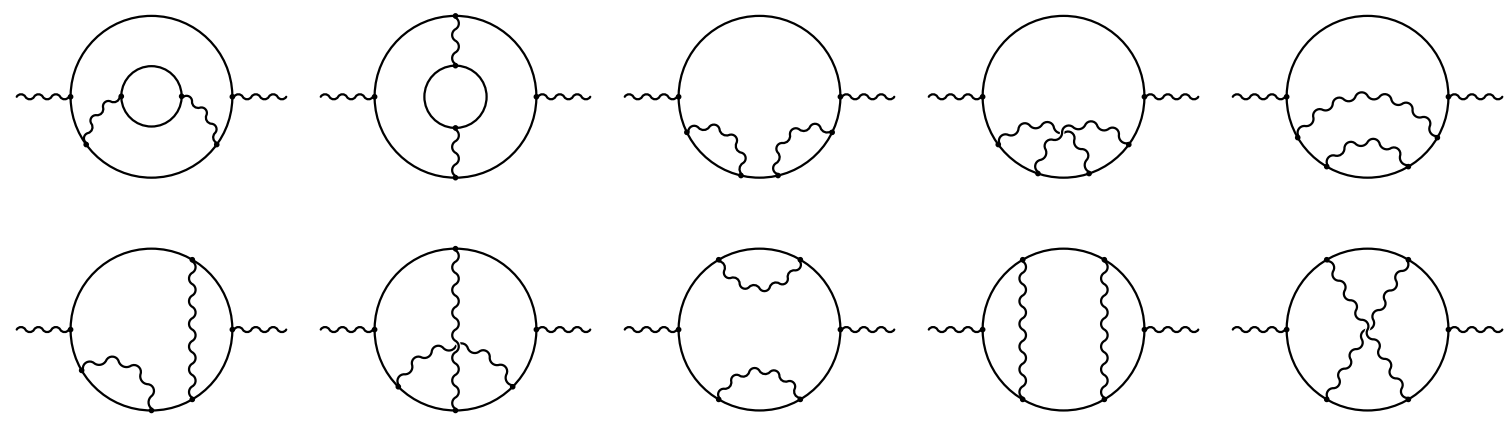

Figure 1. Diagrams contributing to 3-loop photon self energy in QED.

massless lines being cut. The non-polylogarithmic integrals appear only in $(4,0)$ cuts due to the presence of three-loop cut banana graph among the master integrals. Fortunately, all $(4,0)$ cuts are free from divergences of both ultraviolet and infrared origin, and, therefore, can be calculated exactly in $d=4$. In ref. [7] we have presented a set of the master integrals sufficient for this purpose. The master integrals have been expressed via iterated integrals with all weights but one being the rational functions. We have presented also the expressions in terms of one-fold integrals with integrands expressed via complete elliptic integrals and generalized polylogarithms.

We calculate below the master integrals for the remaining cuts $(2,0),(2,1)$, and $(2,2)$. We express the exact results for these cuts via iterated integrals with all weights being rational. These iterated integrals are, therefore, expressible in terms of Goncharov's polylogarithms. Besides the application to the photon self-energy, these master integrals enter the total cross sections of one-photon electron-positron annihilation to muons or to hadrons at NNLO.

For the $(4,0)$ cut we write the results of ref. [7] in terms of similar iterated integrals, with the right-most weight expressed via complete elliptic integrals. We provide explicit expressions for the asymptotics of the obtained integrals, including those from $(4,0)$ class, near thresholds and in the high-energy limit.

Our calculation follows the standard path:

1. IBP reduction. ${ }^{1}$ Constructing differential equations for master integrals.

2. Reduction of the differential equations to $\epsilon$-form.

3. Fixing boundary conditions from threshold asymptotics.

4. Constructing solution in terms of iterated integrals.

\section{Prototypes, LiteRed bases and boundary conditions}

As it was already mentioned in the Introduction, our calculation strategy for polylogarithmic master integrals is based on the reduction of a differential system to $\epsilon$-form $[8,9]$. In

\footnotetext{
${ }^{1}$ For the IBP reduction we use LiteRed package, refs. [11, 12].
} 


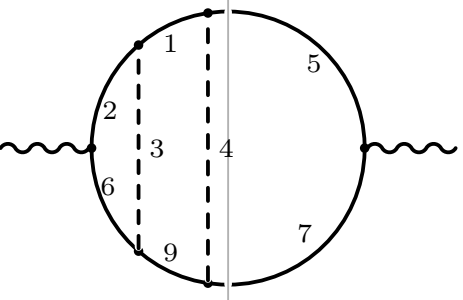

js $[$ cut $20,1,1,1,1,1,1,1,0,1]$

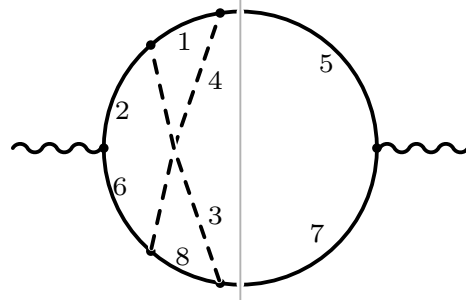

js $[$ cut $20,1,1,1,1,1,1,1,1,0]$

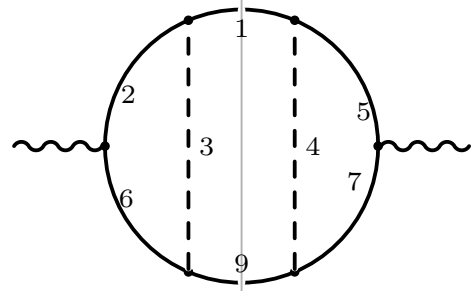

js $[\operatorname{cut} 20 a, 1,1,1,1,1,1,1,0,1]$

Figure 2. Prototypes for master integrals of $(2,0)$ cuts.

the case of finite non-polylogarithmic master integrals we use instead the notion of $\epsilon$-regular basis [7]. In what follows we will use $\boldsymbol{j}$ for the column of LiteRed master integrals and $\boldsymbol{J}$ for the canonical or $\epsilon$-regular basis.

Having reduced the system of differential equations for polylogarithmic master integrals to $\epsilon$-form the latter can be easily solved in terms of Goncharov's polylogarithms and the solution for initial LiteRed master integrals can be written as

$$
\boldsymbol{j}(\beta)=e^{-3 \epsilon \gamma_{E}} T(\beta) \boldsymbol{J}(\beta)=T(\beta) \operatorname{Pexp}\left[\epsilon \int_{0}^{\beta} S(t) d t\right] L \cdot \boldsymbol{c},
$$

where $\beta=\sqrt{1-4 / s}, T$ is the transformation matrix to the canonical basis, and $S$ is the matrix entering differential equations system for the canonical master integrals

$$
\partial_{\beta} \boldsymbol{J}(\beta)=\epsilon S(\beta) \boldsymbol{J}(\beta),
$$

$\boldsymbol{c}$ is the column of the coefficients in threshold asymptotic expansions of the LiteRed master integrals, and $L$ is a rational matrix depending on $\epsilon$.

The reduction to $\epsilon$-form, the choice of the coefficients $\boldsymbol{c}$, and the determination of the corresponding "adapter" matrix $L$ is made with the help of Libra package [10].

It appears that all required families of integrals can be conveniently described using the following nine "denominators":

$$
\begin{array}{llllr}
D_{1}=1-l_{2}^{2}, & D_{2}=1-l_{3}^{2}, & D_{3}=-\left(l_{2}-l_{3}\right)^{2}, & D_{4}=-\left(l_{1}-l_{2}\right)^{2}, & D_{5}=1-l_{1}^{2}, \\
D_{6}=1-\left(q-l_{3}\right)^{2}, & D_{7}=1-\left(q-l_{1}\right)^{2}, & D_{8}=1-\left(q-l_{1}+l_{2}-l_{3}\right)^{2}, & D_{9}=1-\left(q-l_{2}\right)^{2} . & (2.3)
\end{array}
$$

$(2,0)$ cuts. In the case of $(2,0)$ cuts all LiteRed master integrals belong to one of the three prototypes shown in figure 2. As the two first prototypes differ only by one denominator, we use one LiteRed basis for them. Namely, we have

$$
\begin{aligned}
& j_{n_{1} \ldots, n_{9}}^{\text {cut20 }}=\frac{(2 \pi)^{2}}{2 \pi^{d / 2}} \int \frac{d^{d} l_{1} d^{d} l_{2} d^{d} l_{3}}{\left(i \pi^{d / 2}\right)^{2}} \frac{\delta^{\left(n_{5}-1\right)}\left(-D_{5}\right) \delta^{\left(n_{7}-1\right)}\left(-D_{7}\right)}{\prod_{k=1, k \notin\{5,7\}}^{9}\left(D_{k}-i 0\right)^{n_{k}}} \\
& j_{n_{1} \ldots, n_{9}}^{\text {cut20a }}=\frac{(2 \pi)^{2}}{2 \pi^{d / 2}} \int \frac{d^{d} l_{1} d^{d} l_{2} d^{d} l_{3}}{\left(i \pi^{d / 2}\right)\left(-i \pi^{d / 2}\right)} \frac{\delta^{\left(n_{1}-1\right)}\left(-D_{1}\right) \delta^{\left(n_{9}-1\right)}\left(-D_{9}\right)}{\prod_{k=1, k \notin\{1,9\}}^{9}\left(\sigma_{k} D_{k}-i 0\right)^{n_{k}}},
\end{aligned}
$$


where one of $n_{8}, n_{9}$ is necessarily non-positive in the first family and $n_{8} \leqslant 0$ in the second. We also use notation $\sigma_{k}=-1$ if $k \in\{4,5,7\}$ and $\sigma_{k}=1$ otherwise. The denominators are defined in eq. (2.3).

We have 17 master integrals in the first family and 2 additional master integrals in the second:
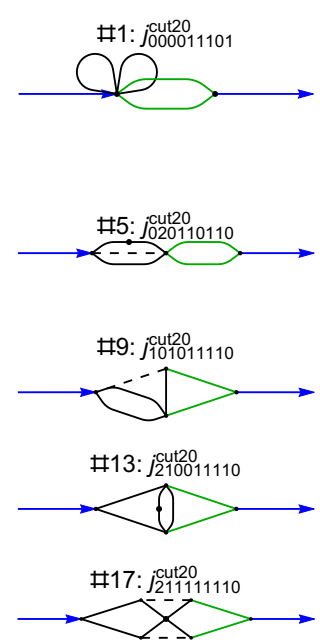
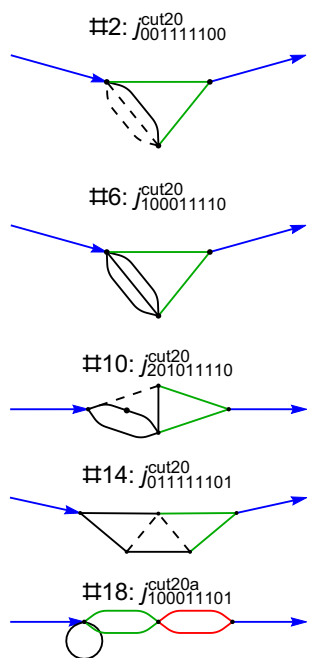
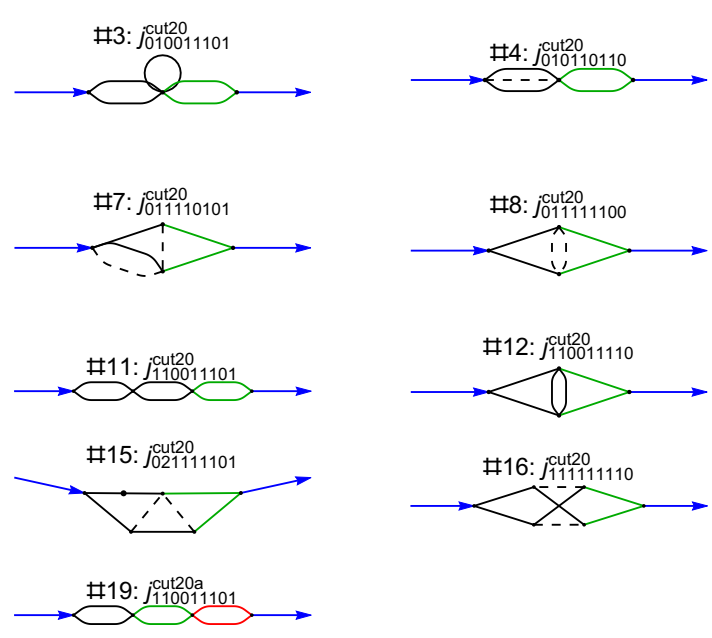

Here the green lines correspond to the cut propagators, while the red lines correspond to complex conjugated part of the diagram. Note that the integral $j_{100011101}^{\text {cut20a }}$ is a complex conjugate of $j_{010011101}^{\text {cut20 }}$ and we introduce it because it enters the differential equation for $j_{110011101}^{\text {cut20a }}$

Using Libra we have chosen the following constants to fix our boundary conditions:

$$
\begin{gathered}
\boldsymbol{c}=\left(c_{1}\left(\beta^{1-2 \epsilon}\right), c_{2}\left(\beta^{1-2 \epsilon}\right), c_{3}\left(\beta^{2-4 \epsilon}\right), c_{4}\left(\beta^{6-8 \epsilon}\right), c_{4}\left(\beta^{1-2 \epsilon}\right), c_{6}\left(\beta^{1-2 \epsilon}\right), c_{7}\left(\beta^{0}\right),\right. \\
c_{8}\left(\beta^{2-6 \epsilon}\right), c_{9}\left(\beta^{0}\right), c_{9}\left(\beta^{3-6 \epsilon}\right), c_{11}\left(\beta^{3-6 \epsilon}\right), c_{12}\left(\beta^{0}\right), c_{12}\left(\beta^{1-4 \epsilon}\right), c_{14}\left(\beta^{0}\right), \\
\left.c_{14}\left(\beta^{1-6 \epsilon}\right), c_{16}\left(\beta^{0}\right), c_{16}\left(\beta^{-1-6 \epsilon}\right), c_{18}\left(\beta^{2-4 \epsilon}\right), c_{19}\left(\beta^{3-6 \epsilon}\right)\right)^{\top},
\end{gathered}
$$

where $c_{i}\left(\beta^{\nu}\right)$ denotes the coefficient in front of $\beta^{\nu}$ in the threshold asymptotics of $i$-th LiteRed master integral. We calculate the required integrals using the expansion-byregions method, ref. [13].

Let us present a few examples of calculation of the required coefficients. First, note that the above master integrals represent either the 2-loop vertex or the product of 1-loop vertices integrated over 2-particle phase space. The later integration decouples completely and we have

$$
\Phi_{2}=\frac{(2 \pi)^{2}}{2} \int \frac{d^{d} l_{1}}{\pi^{d / 2}} \delta\left(l_{1}^{2}-1\right) \delta\left(\left(q-l_{1}\right)^{2}-1\right)=\frac{\pi^{3 / 2}}{2 \Gamma(3 / 2-\epsilon)}\left(\frac{s}{4}\right)^{-\epsilon} \beta^{1-2 \epsilon}
$$

Noting that it scales as $\sim \beta^{1-2 \epsilon}$ we immediately conclude that

$$
c_{7}\left(\beta^{0}\right)=c_{9}\left(\beta^{0}\right)=c_{12}\left(\beta^{0}\right)=c_{14}\left(\beta^{0}\right)=c_{16}\left(\beta^{0}\right)=0 .
$$


To perform the threshold expansion of two-loop vertex integrals we use the expansionby-regions method [13] in momentum space. We first ensure that the routing of hard external momentum goes only through the massive lines of the vertex graph. Then we have four different regions for each loop momentum $l$ : hard $\left(l_{0} \sim 1,|\vec{l}| \sim 1\right)$, soft $\left(l_{0} \sim \beta,|\vec{l}| \sim\right.$ $\beta$ ), potential $\left(l_{0} \sim \beta^{2},|\vec{l}| \sim \beta\right)$ and ultrasoft $\left(l_{0} \sim \beta^{2},|\vec{l}| \sim \beta^{2}\right)$. Each of these regions contributes to some specific powers of the expansion in $\beta$ and thus to some particular coefficient $c_{i}\left(\beta^{\nu}\right)$. For example, consider $c_{14}\left(\beta^{1-6 \epsilon}\right)$ coefficient. In this case the 2-loop vertex part of LiteRed master integral is given by $\left(p_{1}^{2}=p_{2}^{2}=1,\left(p_{1}+p_{2}\right)^{2}=s\right)$ :

$$
\int \frac{d^{d} l_{1} d^{d} l_{2}}{\left(i \pi^{d / 2}\right)^{2}} \frac{1}{\left(-l_{2} \cdot p_{2}-l_{2}^{2}\right)\left(2 l_{1} \cdot p_{1}-l_{1}^{2}\right)\left(2 l_{2} \cdot p_{1}-l_{2}^{2}\right)\left(-l_{1}^{2}\right)\left(-\left(l_{1}-l_{2}\right)^{2}\right)}
$$

and the required region (soft-potential) contributing to $\beta^{1-6 \epsilon}$ power is given by $l_{1,0} \sim$ $\left|\vec{l}_{1}\right| \sim\left|\vec{l}_{2}\right| \sim \beta, l_{2,0} \sim \beta^{2}$. Expanding the integrand in this region to leading power in $\beta$, we express the integrals over $l_{1,0}, l_{2,0}$ as a sum over residues. The remaining integrals over $\vec{l}_{1}$ and $\vec{l}_{2}$ can be conveniently evaluated using alpha-parametrization. Finally, restoring the 2-particle phase space integral we get

$$
c_{14}\left(\beta^{1-6 \epsilon}\right)=\frac{2^{2 \epsilon-1} e^{2 i \pi \epsilon} \pi^{4} \Gamma(-4 \epsilon) \Gamma(2 \epsilon) \sec (\pi \epsilon)}{\Gamma(3 / 2-3 \epsilon) \Gamma(3 / 2-\epsilon) \Gamma(-\epsilon)} .
$$

Similarly, a careful inspection of regions gives

$$
c_{14}\left(\beta^{1-4 \epsilon}\right)=c_{16}\left(\beta^{-1-6 \epsilon}\right)=0 .
$$

It is also instructive to see some details of calculation of $c_{4}\left(\beta^{1-2 \epsilon}\right)$ constant, which appears to be expressible via hypergeometric function ${ }_{3} F_{2}$. The required scaling in $\beta$ in this case is already provided by 2-particle phase space integral and 2-loop vertex part should be evaluated at $\beta=0$, which corresponds to hard-hard region in the threshold expansion. Using alpha parametrization for 2-loop sunset subdiagram we have

$$
\begin{aligned}
c_{4}\left(\beta^{1-2 \epsilon}\right)= & \frac{\pi^{3 / 2} \Gamma(-1+2 \epsilon)}{2 \Gamma(3 / 2-\epsilon)} \int_{0}^{\infty} d \alpha_{2} \int_{0}^{\infty} d \alpha_{3} \int_{0}^{\infty} d \alpha_{6} \delta\left(1-\alpha_{2}-\alpha_{3}-\alpha_{6}\right) \\
& \times\left(\alpha_{6}\left(\alpha_{2}-\alpha_{3}\right)^{2}+\alpha_{2} \alpha_{3}\left(\alpha_{2}+\alpha_{3}\right)\right)^{1-2 \epsilon}\left(\alpha_{3} \alpha_{6}+\alpha_{2}\left(\alpha_{3}+\alpha_{6}\right)\right)^{3(\epsilon-1)} .
\end{aligned}
$$

Using Cheng-Wu theorem [14] we replace $\delta\left(1-\alpha_{2}-\alpha_{3}-\alpha_{6}\right)$ with $\delta\left(1-\alpha_{2}-\alpha_{3}\right)$ and making the change of variables $\alpha_{2}=x, \alpha_{3}=1-x, \alpha_{6}=x(1-x) y$ we obtain

$$
c_{4}\left(\beta^{1-2 \epsilon}\right)=\frac{\pi^{3 / 2} \Gamma(-1+2 \epsilon)}{2 \Gamma(3 / 2-\epsilon)} \int_{0}^{1} d x \int_{0}^{\infty} d y(x(1-x))^{\epsilon-1}(y+1)^{3 \epsilon-3}\left((1-2 x)^{2} y+1\right)^{1-2 \epsilon}
$$



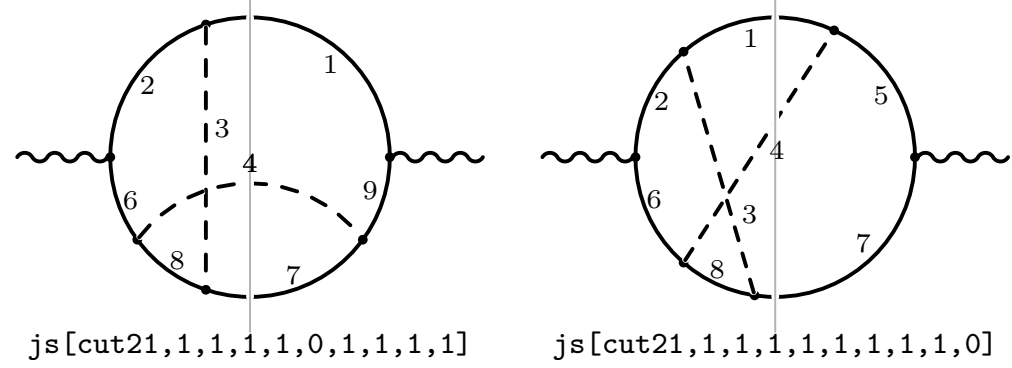

Figure 3. Prototypes for master integrals of $(2,1)$ cuts.

Now we use the Mellin-Barnes parametrization

$$
\frac{\Gamma(-1+2 \epsilon)}{\left((1-2 x)^{2} y+1\right)^{2 \epsilon-1}}=\int_{-i \infty}^{i \infty} \frac{d \tau}{2 \pi i} \frac{\Gamma(-1+2 \epsilon-\tau) \Gamma(\tau) e^{i \pi \tau}}{(1+y)^{-1+2 \epsilon-\tau}(4 y x(1-x))^{\tau}}
$$

After this the integrals over $x$ and $y$ can be taken in terms of $\Gamma$-functions and we obtain one-fold Mellin-Barnes representation

$$
c_{4}\left(\beta^{1-2 \epsilon}\right)=\frac{\pi^{5 / 2}}{2 \Gamma(3 / 2-\epsilon)} \int_{-i \infty}^{i \infty} \frac{d \tau}{2 \pi i} \frac{4^{-\tau} e^{i \pi \tau} \Gamma(1-\epsilon) \Gamma(\epsilon-\tau)^{2} \Gamma(2 \epsilon-\tau-1)}{\sin (\pi \tau) \Gamma(2 \epsilon-2 \tau) \Gamma(-\epsilon-\tau+2)}
$$

We close the contour of integration to the left and finally obtain

$$
c_{4}\left(\beta^{1-2 \epsilon}\right)=\frac{\pi^{5 / 2} 2^{2-4 \epsilon} \Gamma(2 \epsilon-1)}{\sin (\pi \epsilon) \Gamma(3-2 \epsilon) \Gamma\left(\epsilon+\frac{1}{2}\right)}{ }_{3} F_{2}\left(1, \epsilon, 2 \epsilon-1 ; 2-\epsilon, \epsilon+\frac{1}{2} ; 1\right) .
$$

In a similar way, we have been able to fix all required boundary constants exactly in $\epsilon$, with two of them expressed via hypergeometric functions. E.g., we have

$$
c_{6}\left(\beta^{1-2 \epsilon}\right)=\frac{\pi^{2} \Gamma(\epsilon)\left({ }_{3} F_{2}\left(\frac{1}{2}, 1,2 \epsilon-1 ; 2-\epsilon, \epsilon+\frac{1}{2} ; 1\right)+(1-2 \epsilon){ }_{3} F_{2}\left(1, \frac{3}{2}-\epsilon, \epsilon ; \frac{3}{2}, 3-2 \epsilon ; 1\right)\right)}{2^{2 \epsilon-1} \Gamma(3-2 \epsilon)(2 \epsilon-1) \sin (\pi \epsilon)} .
$$

We have checked that a few leading terms of $\epsilon$-expansion of $c_{i}^{a+b \epsilon}$ constants calculated here can be reproduced from the results of 2-loop vertex diagrams calculation presented in [15].

$(2,1)$ cuts. All LiteRed master integrals for $(2,1)$ cuts belong to two prototypes in figure 3 . They are defined as

$$
j_{n_{1} \ldots, n_{9}}^{\mathrm{cut} 21}=\frac{(2 \pi)^{3}}{2\left(\pi^{d / 2}\right)^{2}} \int \frac{d^{d} l_{1} d^{d} l_{2} d^{d} l_{3}}{i \pi^{d / 2}} \frac{\prod_{k \in\{1,4,7\}} \delta^{\left(n_{k}-1\right)}\left(-D_{k}\right)}{\prod_{k=1, k \notin\{1,4,7\}}^{9}\left(D_{k}-i 0\right)^{n_{k}}},
$$


where one of $n_{5}, n_{9}$ is necessarily non-positive and $D_{k}$ are defined in eq. (2.3). We have 22 master integrals:
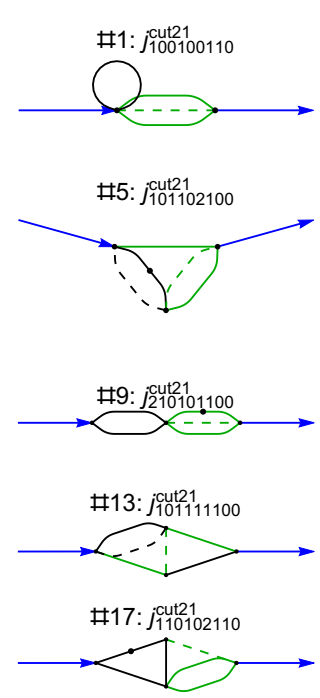

\#21: jut21 $\int_{11111110}^{\text {con }}$
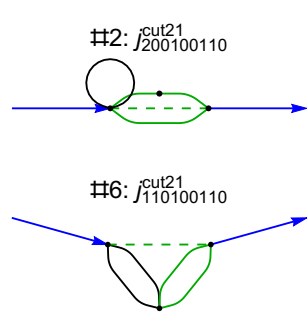

\#10: $j_{101101110}^{\text {cut21 }}$

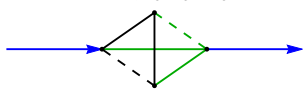

\#14: $j_{110100111}^{\text {cut21 }}$

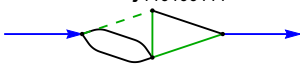

\#18: jut111 $j_{11101100}$

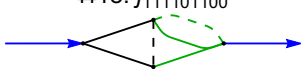

\#22: $j_{121111110}^{\text {cut21 }}$
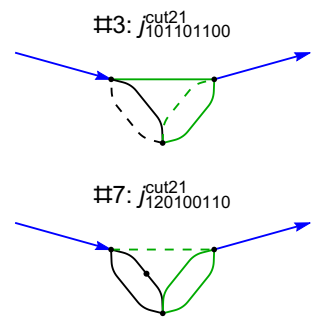

\#11: jut21 $j_{101102110}$

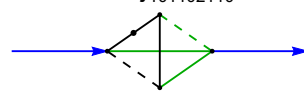

\#15: j: j20121

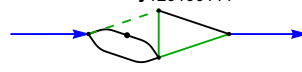

\#19: jut21 j1111100

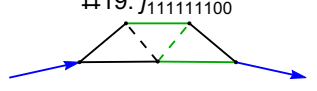

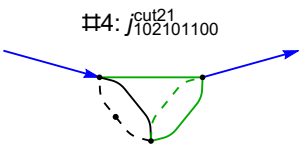

\#8: jut21 $j_{110101100}^{\text {cut }}$

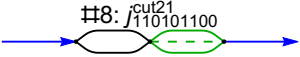

\#12: $j_{2011011110}^{\text {cut21 }}$

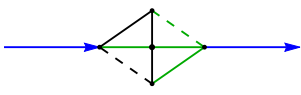

\#16: jut121 $j_{10101110}^{\text {cut }}$

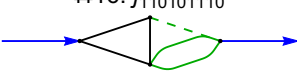

\#20: $j_{121111100}^{\text {cut21 }}$

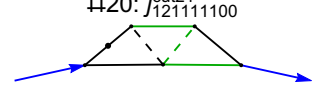

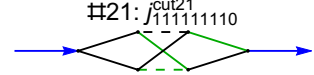

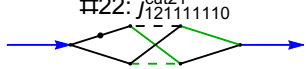

We fix boundary conditions by calculating the following coefficients in the threshold asymptotics of the LiteRed master integrals:

$$
\begin{aligned}
\boldsymbol{c}=( & c_{1}\left(\beta^{0}\right), c_{1}\left(\beta^{5-6 \epsilon}\right), c_{3}\left(\beta^{0}\right), c_{3}\left(\beta^{2}\right), c_{3}\left(\beta^{7-10 \epsilon}\right), c_{6}\left(\beta^{0}\right), c_{6}\left(\beta^{6-8 \epsilon}\right), c_{8}\left(\beta^{6-8 \epsilon}\right), \\
& c_{8}\left(\beta^{1-2 \epsilon}\right), c_{10}\left(\beta^{0}\right), c_{10}\left(\beta^{2}\right), c_{10}\left(\beta^{3-6 \epsilon}\right), c_{13}\left(\beta^{0}\right), c_{14}\left(\beta^{0}\right), c_{15}\left(\beta^{1-6 \epsilon}\right), \\
& \left.c_{16}\left(\beta^{0}\right), c_{16}\left(\beta^{3-6 \epsilon}\right), c_{18}\left(\beta^{0}\right), c_{19}\left(\beta^{0}\right), c_{20}\left(\beta^{-6 \epsilon-1}\right), c_{21}\left(\beta^{0}\right), c_{21}\left(\beta^{-6 \epsilon-1}\right)\right)^{\top} .
\end{aligned}
$$

among which only four are nonzero:

$$
\begin{array}{ll}
c_{1}\left(\beta^{5-6 \epsilon}\right)=\frac{\pi^{5 / 2} 2^{1-2 \epsilon} \csc (\pi \epsilon)}{(\epsilon-1) \Gamma\left(\frac{7}{2}-3 \epsilon\right)}, & c_{3}\left(\beta^{7-10 \epsilon}\right)=\frac{\pi^{2} 2^{1-2 \epsilon} e^{2 i \pi \epsilon} \epsilon \Gamma(3-4 \epsilon) \Gamma(-\epsilon) \Gamma(2 \epsilon-1)}{\Gamma\left(\frac{9}{2}-5 \epsilon\right) \Gamma\left(\frac{3}{2}-\epsilon\right)}, \\
c_{6}\left(\beta^{6-8 \epsilon}\right)=\frac{\pi^{3 / 2} 2^{1-4 \epsilon} e^{i \pi \epsilon} \Gamma(1-\epsilon)^{2} \Gamma\left(\epsilon-\frac{1}{2}\right)}{i \Gamma(4-4 \epsilon)}, & c_{8}\left(\beta^{6-8 \epsilon}\right)=\frac{\pi^{2} 4^{-\epsilon} e^{i \pi \epsilon} \Gamma(1-\epsilon) \Gamma\left(\epsilon-\frac{1}{2}\right)}{i \Gamma\left(\frac{7}{2}-3 \epsilon\right)} .
\end{array}
$$

It is remarkable that the differential equations for $(2,1)$-cut master integrals are the only ones which have singularity at $s=1$. Consequently the $(2,1)$-cut master integrals can not be expressed via harmonic polylogarithms because they involve Goncharov's polylogarithms with letters $\pm \sqrt{3} i$.

$(2,2)$ cuts. All LiteRed master integrals for $(2,2)$ cuts belong to three prototypes in figure 4. They are defined via two LiteRed bases

$$
\begin{aligned}
& j_{n_{1} \ldots, n_{9}}^{\text {cut22 }}=\frac{(2 \pi)^{4}}{2\left(\pi^{d / 2}\right)^{3}} \int d^{d} l_{1} d^{d} l_{2} d^{d} l_{3} \frac{\prod_{k \in\{1,3,4,8\}} \delta^{\left(n_{k}-1\right)}\left(-D_{k}\right)}{\prod_{k=1, k \notin\{1,3,4,8\}}^{9}\left(D_{k}-i 0\right)^{n_{k}}}, \\
& j_{n_{1} \ldots, n_{9}}^{\text {cut22a }}=\frac{(2 \pi)^{4}}{2\left(\pi^{d / 2}\right)^{3}} \int d^{d} l_{1} d^{d} l_{2} d^{d} l_{3} \frac{\prod_{k \in\{3,4,5,6\}} \delta^{\left(n_{k}-1\right)}\left(-D_{k}\right)}{\prod_{k=1, k \notin\{3,4,5,6\}}^{9}\left(D_{k}-i 0\right)^{n_{k}}},
\end{aligned}
$$




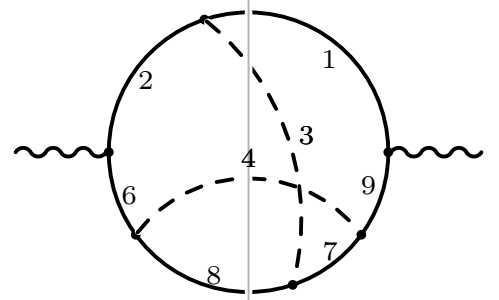

js $[\operatorname{cut} 22,1,1,1,1,0,1,1,1,1]$

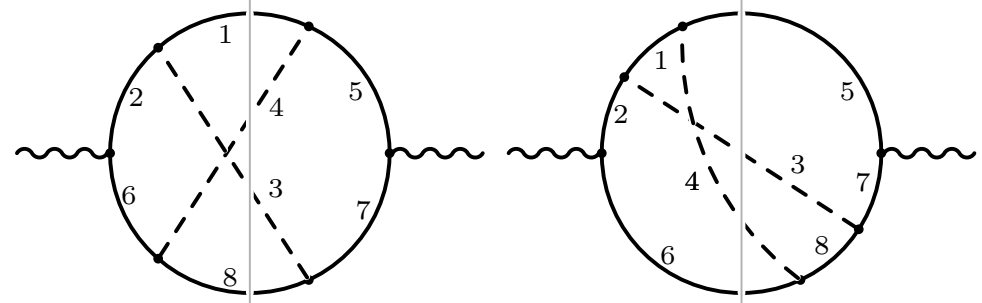

js $[\operatorname{cut} 22,1,1,1,1,1,1,1,1,0] \quad j s[\operatorname{cut} 22 a, 1,1,1,1,1,1,1,1,0]$

Figure 4. Prototypes for master integrals of $(2,2)$ cuts.

where one of $n_{5}, n_{9}$ is necessarily non-positive for the first basis and $n_{9} \leqslant 0$ for the second. The functions $D_{k}$ are defined in eq. (2.3). We have 15 master integrals:

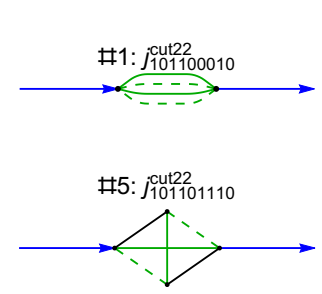

\#9: jout22 11111110

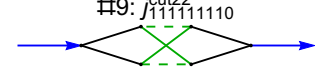

\#13: $j_{021111110}^{\text {cut22a }}$
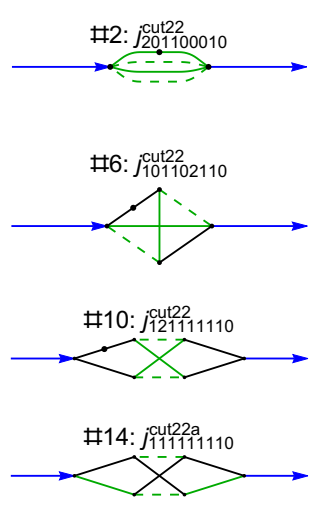
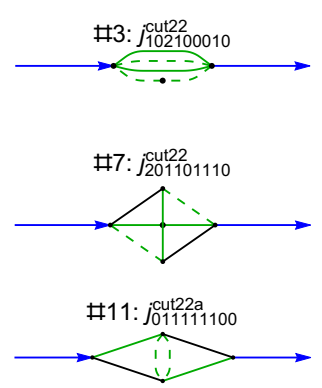

\#15: jut22a
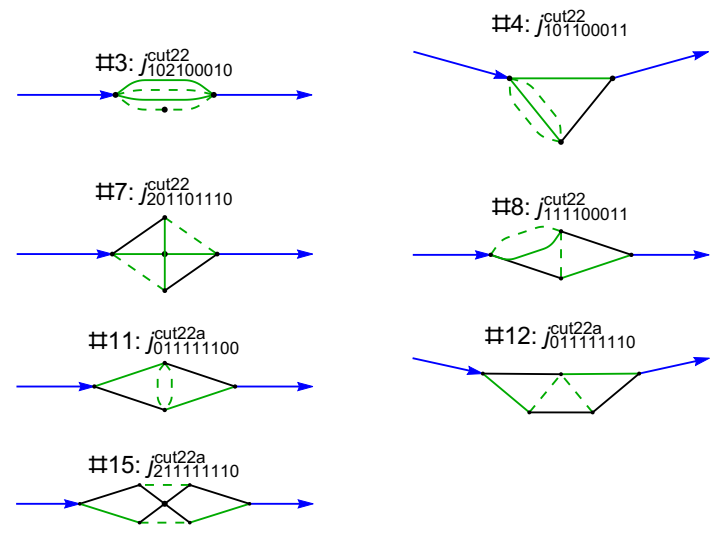

We fix boundary conditions by calculating the following coefficients in the threshold asymptotics of the LiteRed master integrals:

$$
\begin{gathered}
\boldsymbol{c}=\left(c_{1}\left(\beta^{0}\right), c_{1}\left(\beta^{2}\right), c_{1}\left(\beta^{9-10 \epsilon}\right), c_{4}\left(\beta^{1-2 \epsilon}\right), c_{5}\left(\beta^{0}\right), c_{5}\left(\beta^{2}\right), c_{5}\left(\beta^{3-6 \epsilon}\right), c_{8}\left(\beta^{0}\right), c_{9}\left(\beta^{0}\right),\right. \\
\left.c_{9}\left(\beta^{-6 \epsilon-1}\right), c_{11}\left(\beta^{2-6 \epsilon}\right), c_{12}\left(\beta^{0}\right), c_{12}\left(\beta^{1-6 \epsilon}\right), c_{14}\left(\beta^{0}\right), c_{14}\left(\beta^{-6 \epsilon-1}\right)\right)^{\top} .
\end{gathered}
$$

The only nonzero constant is

$$
c_{1}\left(\beta^{9-10 \epsilon}\right)=-\frac{2 \pi^{7 / 2} \csc (2 \pi \epsilon) \Gamma(2-2 \epsilon)}{\Gamma\left(\frac{11}{2}-5 \epsilon\right) \Gamma\left(\frac{3}{2}-\epsilon\right)^{2} \Gamma(2 \epsilon-1)} .
$$

$(4,0)$ cuts. All LiteRed master integrals for $(4,0)$ cuts belong to two prototypes in figure 5 . They are defined as

$$
j_{n_{1} \ldots, n_{9}}^{\mathrm{cut} 40}=\frac{(2 \pi)^{4}}{2\left(\pi^{d / 2}\right)^{3}} \int d^{d} l_{1} d^{d} l_{2} d^{d} l_{3} \frac{\prod_{k \in\{1,6,7,8\}} \delta^{\left(n_{k}-1\right)}\left(-D_{k}\right)}{\prod_{k=1, k \notin\{1,6,7,8\}}^{9}\left(D_{k}-i 0\right)^{n_{k}}},
$$



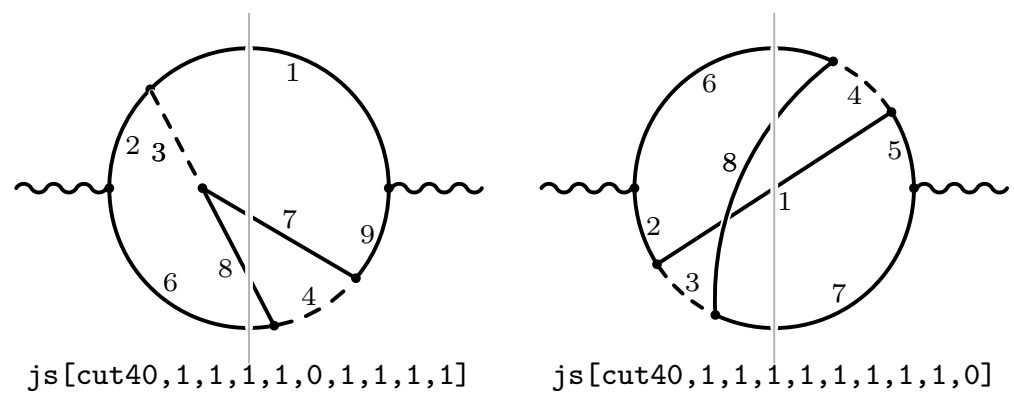

js $[\operatorname{cut} 40,1,1,1,1,0,1,1,1,1]$

Figure 5. Prototypes for master integrals of $(4,0)$ cuts.

where one of $n_{5}, n_{9}$ is necessarily non-positive. We find 13 master integrals:

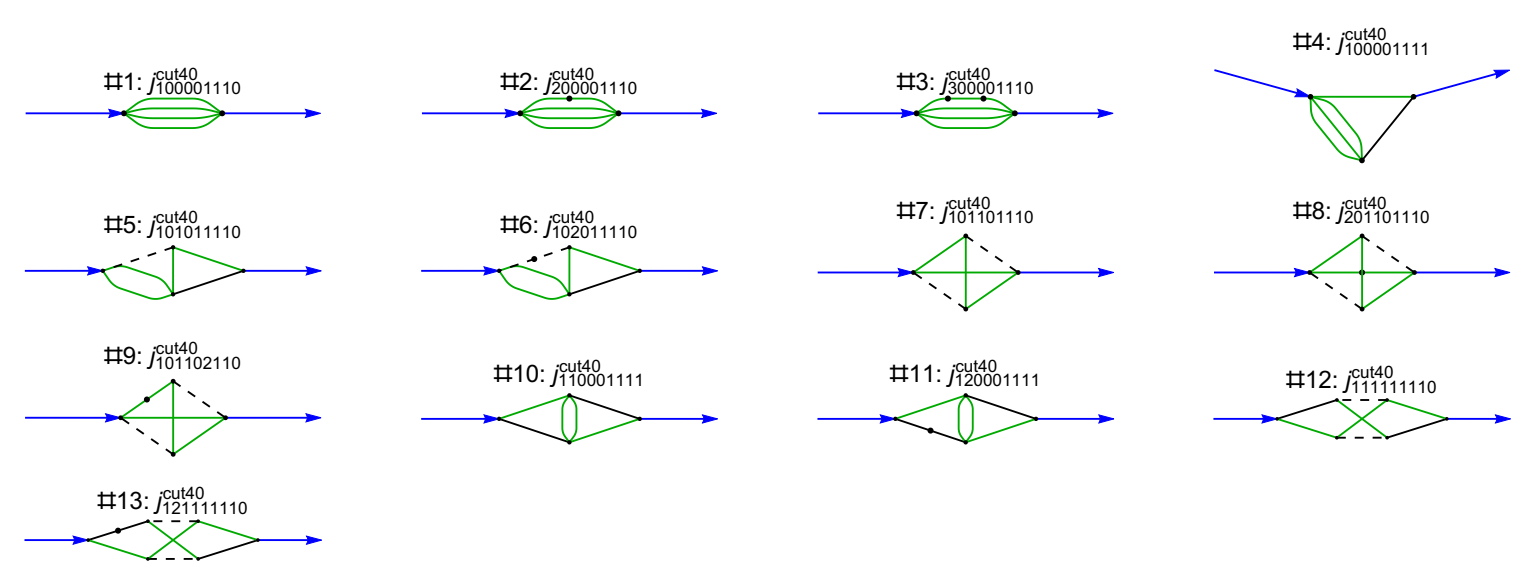

Boundary conditions are fixed by the threshold asymptotics of the first integral (phase space of four massive particles).

\section{Results}

The results of our calculation in its full length are presented in the supplementary material attached to this paper. Let us describe briefly the content of these files:

Data/weights - file with definition of weights. The function $f$ entering the some weights is defined in eq. (A.4) (see description of Data/frules40 below).

Data/jtoGraph $k l$ - file containing graphs for the LiteRed master integrals for the cut $(k, l)$.

Data/jtoJkl - substitution rules for the LiteRed master integrals in terms of the canonical/ $\epsilon$-regular basis.

Data/M $k l$ - matrix in the right-hand side of the differential system $\partial_{s} \boldsymbol{J}=M \boldsymbol{J}$.

Data/T $k l$ - transformation matrix to $\epsilon$-form $/ \epsilon$-regular basis.

cskl - substitution rules for the coefficients of threshold asymptotic expansion used to fix the boundary conditions. 
Data/JtoII $k l$ - substitution rules for the canonical/ $\epsilon$-regular basis in terms of the iterated integrals defined below.

Data/Jthrkl - substitution rules for the threshold asymptotics of the canonical/ $\epsilon$-regular basis. Note that the $(4,0)$ cut has threshold at $s=16$, in contrast to $(2, l)$ cuts.

Data/Jhigh $k l$ - substitution rules for the high-energy asymptotics of the canonical/ $\epsilon$ regular basis.

Data/IItoG - file containing substitution rules for the iterated integrals in terms of Goncharov's polylogarithms.

Data/JtoN40 - file containing the substitution rules for the numerical evaluation of the non-polylogarithmic master integrals for $(4,0)$ cut.

Data/IIrtoLeft40 - "unshuffling" rules for moving $r_{0}, r_{1}, r_{2}, r_{3}$, and $\tilde{r}_{3}$ to the left-most position.

Data/frules40 - definition of function $f$, eq. (A.4), and its differentiation rule.

masters-results.nb - this is the exemplary Mathematica notebook showing the usage of the above data files.

Note that in the supplementary material we have chosen to present the $\epsilon$-expansions of the canonical/ $\epsilon$-regular master integrals rather than those of LiteRed master integrals. The reason is that it appears to be beneficial to first substitute in the cross section (or spectral density) the later via the former and then to substitute the expansions for canonical/ $\epsilon$-regular integrals. Doing it this way one avoids unnecessary loss of the higher orders in $\epsilon$.

One more remark is in place here. In most physical applications the master integrals calculated in the present paper enter in the sum with their mirrored counterpart, which corresponds to complex conjugation. Therefore, only the real part of the results presented here is relevant for such applications (spectral density is one of them).

For expository and reference purposes we present the leading terms of $\epsilon$-expansion of the LiteRed master integrals in the appendix.

\section{Asymptotics}

Now let us describe in some details the calculation of the threshold and high energy asymptotics of the master integrals. The polylogarithmic master integrals for the $(2,0),(2,1)$ and $(2,2)$ cuts were expressed in terms of Goncharov's polylogarithms of argument $\beta$ and thus the calculation of their asymptotic expansions proceeds along the same lines. At threshold ( $s=4$ or $\beta=0)$ the expansion is particularly simply. All one needs to do is to expand the integrand in a power series and integrate term-wise. The corresponding results up to $O\left(\beta^{5}\right)$ can be found in the accompanying files Jthr2*. To obtain the expansion in the high 
energy limit we first rewrite polylogarithms in $\beta$ in terms of polylogarithms of argument $1-\beta$ using recursively the following transformation

$$
G\left(a_{1}, \ldots, a_{k}, \beta\right)=G\left(a_{1}, \ldots, a_{k}, 1\right)+\int_{0}^{1-\beta} \frac{d t}{t-\left(1-a_{1}\right)} G\left(a_{2}, \ldots, a_{k}, 1-t\right) .
$$

To expand the resulting polylogarithms of argument $1-\beta$ we again expand under the integral sign. The high-energy expansions up $O\left(1 / s^{5}\right)$ can be found in accompanying files Jhigh2*.

The asymptotic expansions for non-polylogarithmic master integrals for $(4,0)$ are calculated somewhat differently. First, we use the shuffling relations to rewrite the expressions for master integrals in terms of iterated integrals with transcendental kernels appearing only in the last integration. The inner integrals are then expressed via classical polylogarithms. ${ }^{2}$ As a particular example let us consider the following element of $\epsilon$-regular basis:

$$
J_{06}^{\text {cut } 40}(s)=\frac{1}{2} I\left(l_{2} \tilde{r}_{3} \mid s\right)-I\left(r_{3} \mid s\right)
$$

Using the shuffling relations we move the transcendental weights $r_{k}$ to the left:

$$
J_{06}^{\text {cut } 40}(s)=-I\left(r_{3} \mid s\right)+\frac{1}{2} I\left(l_{2} \mid s\right) I\left(\tilde{r}_{3} \mid s\right)-\frac{1}{2} I\left(\tilde{r}_{3}, l_{2} \mid s\right)
$$

and rewrite the inner polylogarithmic integrals in terms of classical polylogarithms:

$$
J_{06}^{\text {cut } 40}(s)=-I\left(r_{3} \mid s\right)-\frac{1}{2} \ln \left(\frac{12}{s-4}\right) I\left(\tilde{r}_{3} \mid s\right)+\frac{1}{2} I\left(\tilde{r}_{3} \ln \left(\frac{12}{s-4}\right) \mid s\right) .
$$

Now, we have a one-fold integral representation, whose threshold (at $s=16$ ) asymptotics can be easily found by Taylor expanding its integrand at $s=16$ and performing trivial integration. To find its high-energy asymptotics it is convenient to use the identity ${ }^{3}$

$$
\int_{16}^{s} d s f(s)=\int_{16}^{\infty} d s f(s)-\int_{s}^{\infty} d s f(s)
$$

The first term here is some constant which is difficult to determine by explicit analytical evaluation of the integral. Therefore, we use the numerical approach to recognize this constant as a rational combination of (alternating) multiple zeta values using PSLQ algorithm [18]. In the second term we can safely perform Taylor expansion of the integrand at large $s$ and take the remaining integral term-wise. Note that this approach requires to recognize exactly one constant for each integral, independently on the chosen expansion order in $1 / s$. The asymptotics of other master integrals can be computed along the same lines and the results can be found in the accompanying files $\operatorname{Jthr}(4,0)$ and $\operatorname{Jhigh}(4,0)$.

We have checked that the obtained asymptotic expansions are consistent with numerical values of the corresponding integrals in the threshold and the high-energy regions.

\footnotetext{
${ }^{2}$ For this purpose we use the HPL package $[16,17]$.

${ }^{3}$ Note that this identity is not literally applicable to most of the integrals under consideration because the first term in eq. (4.5) diverges at large $s$. In this case we subtract from the integrand several terms of its high-energy asymptotics.
} 


\section{Conclusion}

In this paper we have presented the results for the master integrals entering the three-loop spectral density of photon self energy in QED. The non-polylogarithmic master integrals have been considered earlier in our work [7]. Here we have calculated the remaining integrals which are expressible via Goncharov's polylogarithms. We used the differential equation method [19-23] and the possibility to reduce the corresponding differential systems to $\epsilon$ form $[8,9]$. Given the available algorithms and tools, in particular, the recently published Mathematica package Libra [10], the reduction to $\epsilon$-form appears to be very simple. We have chosen to fix the boundary conditions at the threshold point. We used Libra to determine which asymptotic coefficients have to be calculated in order to fix the boundary conditions. A somewhat more involved step was the calculation of required coefficients using the expansion by regions method [13]. We have managed to calculate all required coefficients exactly in $\epsilon$. This fact, given the found transformations to $\epsilon$ form, allows one to easily obtain even more terms of $\epsilon$ expansion if needed. To verify obtained expressions we used numerical approach based on sector decomposition method [24-30] as implemented in Fiesta [31].

This approach can be applied directly for the master integrals with $(2,0)$ cuts since they can be easily related to the onshell vertex diagrams. In the case of $(2,1)$ and $(2,2)$ cuts the existing implementations can not be applied directly due to the presence of infrared divergences related to phase-space integration and necessity to perform the resolution of singularities also for these integration variables. Fortunately, this difficulty can be avoided by considering integrals in sufficiently high dimensions, ${ }^{4}$ where the integrals become infraredfinite. The original integrals are then obtained with the use of dimensional recurrence relations [32]. Note, that in the case of $(2,2)$ cuts there are no ultraviolet divergent subgraphs and the sector decomposition is not required at all. In the case of $(2,1)$ cuts the corresponding divergence in parametric representation for one-loop subgraph is factorizes in terms of the external $\Gamma$-function factor while parametric integral itself is convergent. So, in this case sector decomposition is not required also. In addition, to check the results for master integrals with $(2,0)$ cuts we have reproduced the 2-loop vertex diagrams calculation as presented in [15]. Finally, we have presented results for the threshold and high-energy asymptotics of the considered master integrals, including non-polylogarithmic master integrals with $(4,0)$ cuts, and checked their consistency with the obtained exact results.

\section{Acknowledgments}

This work was supported by Russian Science Foundation, grant 20-12-00205.

\footnotetext{
${ }^{4}$ We use $d=6-2 \epsilon$ and $d=8-2 \epsilon$
} 


\section{A Leading terms of $\epsilon$-expansion for LiteRed master integrals}

We introduce the following weights

$$
\begin{aligned}
& l_{0}(s)=\frac{1}{s}, \quad l_{1}(s)=\frac{1}{s \beta}, \quad l_{2}(s)=\frac{1}{s-4}, \quad l_{4}(s)=\frac{1}{s-1}, \\
& r_{k}(s)=\frac{f(s)}{s \beta^{k}} \theta(s-16) \quad(k=0,1,2,3), \\
& \tilde{r}_{3}(s)=\frac{8 \beta(s+2) f(s)}{(s-16)(s-4)^{2}} \theta(s-16),
\end{aligned}
$$

where

$$
\begin{aligned}
f(s) & =\frac{16(s-16)}{s}\left[\mathrm{~K}\left(1-k_{-}\right) \mathrm{K}\left(k_{+}\right)-\mathrm{K}\left(k_{-}\right) \mathrm{K}\left(1-k_{+}\right)\right], \\
k_{ \pm} & =\frac{1}{2}\left[1 \pm\left(1-\frac{8}{s}\right) \sqrt{1-\frac{16}{s}}+\frac{16}{s} \sqrt{1-\frac{4}{s}}\right]
\end{aligned}
$$

and the iterated integrals

$$
I\left(w_{n}, \ldots w_{1} \mid s\right)=\int_{s>s_{n}>\ldots>s_{1}>4} \prod_{k=1}^{n} d s_{k} w_{k}\left(s_{k}\right) .
$$

\section{$(2,0)$ cuts}

$$
\begin{aligned}
& j_{000011101}^{\text {cut20 }}=\frac{\pi \beta}{\epsilon^{2}}+O\left(\frac{1}{\epsilon}\right), \\
& j_{001111100}^{\text {cut20 }}=-\frac{\pi \beta}{2 \epsilon^{2}}+O\left(\frac{1}{\epsilon}\right), \\
& j_{010011101}^{\text {cut20 }}=-\frac{\pi \beta}{\epsilon^{2}}+O\left(\frac{1}{\epsilon}\right), \\
& j_{010110110}^{\text {cut220 }}=-\frac{\pi \beta}{\epsilon^{2}}+O\left(\frac{1}{\epsilon}\right), \\
& j_{020110110}^{\text {cut20 }}=\frac{\pi \beta}{2 \epsilon^{2}}+O\left(\frac{1}{\epsilon}\right) \text {, } \\
& j_{100011110}^{\text {cut20 }}=-\frac{3(\pi \beta)}{2 \epsilon^{2}}+O\left(\frac{1}{\epsilon}\right), \\
& j_{011110101}^{\text {cut20 }}=\frac{\pi \beta}{2 \epsilon^{2}}+O\left(\frac{1}{\epsilon}\right) \text {, } \\
& j_{011111100}^{\text {cut20 }}=\frac{\pi \beta}{2 \epsilon^{2}}+O\left(\frac{1}{\epsilon}\right), \\
& j_{101011110}^{\text {cut20 }}=\frac{\pi \beta}{2 \epsilon^{2}}+O\left(\frac{1}{\epsilon}\right) \text {, } \\
& j_{201011110}^{\mathrm{cut} 20}=\frac{2 \pi^{3} I\left(l_{1} \mid s\right)}{3 s}+\frac{2 i \pi^{2} I\left(l_{1}, l_{1} \mid s\right)}{s}-\frac{2 \pi I\left(l_{1}, l_{1}, l_{1} \mid s\right)}{s}+O\left(\epsilon^{1}\right), \\
& j_{110011101}^{\text {cut20 }}=\frac{\pi \beta}{\epsilon^{2}}+O\left(\frac{1}{\epsilon}\right) \text {, } \\
& j_{110011110}^{\text {cut20 }}=\frac{\pi \beta}{2 \epsilon^{2}}+O\left(\frac{1}{\epsilon}\right) \text {, } \\
& j_{210011110}^{\text {cut20 }}=\frac{\pi^{3} I\left(l_{1} \mid s\right)}{3 s}+\frac{i \pi^{2} I\left(l_{1}, l_{1} \mid s\right)}{s}-\frac{\pi I\left(l_{1}, l_{1}, l_{1} \mid s\right)}{s}+O\left(\epsilon^{1}\right), \\
& j_{011111101}^{\text {cut20 }}=\frac{i \pi\left(2 \pi^{3}+2 i \pi^{2} \ln 2+21 i \zeta_{3}\right) I\left(l_{1} \mid s\right)}{2 s}+\frac{2 \pi^{3} I\left(l_{0}, l_{1} \mid s\right)}{3 s}-\frac{\pi^{3} I\left(l_{1}, l_{0} \mid s\right)}{2 s}+\frac{\pi^{3} I\left(l_{1}, l_{1} \mid s\right)}{s}-\frac{\pi^{3} I\left(l_{1}, l_{2} \mid s\right)}{s} \\
& +\frac{2 i \pi^{2} I\left(l_{0}, l_{1}, l_{1} \mid s\right)}{s}-\frac{i \pi^{2} I\left(l_{1}, l_{0}, l_{1} \mid s\right)}{s}+\frac{i \pi^{2} I\left(l_{1}, l_{1}, l_{2} \mid s\right)}{s}-\frac{2 i \pi^{2} I\left(l_{1}, l_{2}, l_{1} \mid s\right)}{s}-\frac{2 \pi I\left(l_{0}, l_{1}, l_{1}, l_{1} \mid s\right)}{s} \\
& +\frac{\pi I\left(l_{1}, l_{0}, l_{1}, l_{1} \mid s\right)}{s}-\frac{\pi I\left(l_{1}, l_{1}, l_{2}, l_{1} \mid s\right)}{s}+\frac{2 \pi I\left(l_{1}, l_{2}, l_{1}, l_{1} \mid s\right)}{s}+O\left(\epsilon^{1}\right), \\
& j_{021111101}^{\mathrm{cut} 20}=\frac{1}{\epsilon}\left(\frac{i \pi^{2} I\left(l_{1} \mid s\right) \beta}{(s-4) s}-\frac{\pi I\left(l_{1}, l_{1} \mid s\right) \beta}{(s-4) s}+\frac{\pi^{3} \beta}{2(s-4) s}\right)+O\left(\epsilon^{0}\right),
\end{aligned}
$$




$$
\begin{aligned}
j_{111111110}^{\text {cut20 }}= & \frac{1}{\epsilon}\left(\frac{\pi^{2} \beta(\pi+2 i \ln 2) I\left(l_{1} \mid s\right)}{(s-4) s}+\frac{i \pi^{2} \beta I\left(l_{1}, l_{0} \mid s\right)}{(s-4) s}+\frac{i \pi^{2} \beta I\left(l_{1}, l_{2} \mid s\right)}{(s-4) s}-\frac{\pi \beta I\left(l_{1}, l_{0}, l_{1} \mid s\right)}{(s-4) s}-\frac{\pi \beta I\left(l_{1}, l_{2}, l_{1} \mid s\right)}{(s-4) s}\right) \\
& +O\left(\epsilon^{0}\right), \\
j_{211111110}^{\text {cut20 }}= & \frac{1}{\epsilon}\left(\frac{i \pi^{2} I\left(l_{0} \mid s\right)}{2 s^{2}}+\frac{i \pi^{2} I\left(l_{2} \mid s\right)}{2 s^{2}}-\frac{\pi I\left(l_{0}, l_{1} \mid s\right)}{2 s^{2}}+\frac{i \pi^{2} \beta I\left(l_{1}, l_{0} \mid s\right)}{(s-4) s^{2}}+\frac{i \pi^{2} \beta I\left(l_{1}, l_{2} \mid s\right)}{(s-4) s^{2}}-\frac{\pi I\left(l_{2}, l_{1} \mid s\right)}{2 s^{2}}\right. \\
& -\frac{\pi \beta I\left(l_{1}, l_{0}, l_{1} \mid s\right)}{(s-4) s^{2}}-\frac{\pi \beta I\left(l_{1}, l_{2}, l_{1} \mid s\right)}{(s-4) s^{2}}+\frac{\pi\left(-\beta s^{2}-4 \beta s+4 i \pi \ln 2 s+2 \pi^{2} s-8 i \pi s-16 i \pi \ln 2-8 \pi^{2}+16 i \pi\right)}{4(s-4) s^{2}} \\
& \left.+\frac{\pi I\left(l_{1} \mid s\right)\left(2 s+\pi^{2} \beta+2 i \pi \beta \ln 2-4\right)}{(s-4) s^{2}}\right)+O\left(\epsilon^{0}\right), \\
j_{100011101}^{\text {cut20a }}= & -\frac{\pi \beta}{\epsilon^{2}}+O\left(\frac{1}{\epsilon}\right), \\
j_{110011101}^{\text {cut20a }}= & \frac{\pi \beta}{\epsilon^{2}}+O\left(\frac{1}{\epsilon}\right)
\end{aligned}
$$

\section{$(2,1)$ cuts}

$$
\begin{aligned}
& j_{100100110}^{\text {cut } 21}=\frac{1}{\epsilon}\left(\frac{2 \pi(s-1) I\left(l_{1} \mid s\right)}{s}-\frac{1}{2} \pi(s+2) \beta\right)+O\left(\epsilon^{0}\right), \\
& j_{200100110}^{\text {cut } 21}=\frac{1}{\epsilon}\left(\pi \beta-\frac{\pi(s-2) I\left(l_{1} \mid s\right)}{s}\right)+O\left(\epsilon^{0}\right), \\
& j_{101101100}^{\text {cut21 }}=\frac{1}{\epsilon}\left(\frac{1}{2} \pi(s+2) \beta-\frac{2 \pi(s-1) I\left(l_{1} \mid s\right)}{s}\right)+O\left(\epsilon^{0}\right), \\
& j_{102101100}^{\text {cut } 21}=\frac{1}{\epsilon}\left(\frac{\pi(s-2) I\left(l_{1} \mid s\right)}{s}-\pi \beta\right)+O\left(\epsilon^{0}\right), \\
& j_{101102100}^{\text {cut21 }}=\pi I\left(l_{0} \mid s\right) \beta+2 \pi I\left(l_{2} \mid s\right) \beta+\pi(-1-2 i \pi+2 \ln 2) \beta+\frac{\pi s I\left(l_{0}, l_{1} \mid s\right)}{2(s-1)} \\
& -\frac{\pi\left(s^{2}-2 s+4\right) I\left(l_{1}, l_{0} \mid s\right)}{2(s-1) s}-\frac{\pi\left(s^{2}-2 s+4\right) I\left(l_{1}, l_{2} \mid s\right)}{(s-1) s} \\
& +\frac{\pi I\left(l_{1} \mid s\right)\left(-\ln 2 s^{2}+i \pi s^{2}-2 s^{2}+2 \ln 2 s-2 i \pi s+4 s-4 \ln 2+4 i \pi-2\right)}{(s-1) s}+O\left(\epsilon^{1}\right), \\
& j_{110100110}^{\text {cut } 21}=\frac{1}{\epsilon}\left(\frac{1}{2} \pi(s+2) \beta-\frac{2 \pi(s-1) I\left(l_{1} \mid s\right)}{s}\right)+O\left(\epsilon^{0}\right), \\
& j_{120100110}^{\text {cut21 }}=-\frac{i \pi(-i \beta s+\pi s-4 \pi)}{s}+i \pi^{2} I\left(l_{0} \mid s\right)+\frac{\pi(s-2) I\left(l_{1} \mid s\right)}{s}-\pi I\left(l_{0}, l_{1} \mid s\right)+O\left(\epsilon^{1}\right), \\
& j_{110101100}^{\text {cut } 21}=\frac{1}{\epsilon}\left(\frac{1}{2} \pi(s+2) \beta-\frac{2 \pi(s-1) I\left(l_{1} \mid s\right)}{s}\right)+O\left(\epsilon^{0}\right), \\
& j_{210101100}^{\text {cut } 21}=\frac{1}{\epsilon}\left(\frac{\pi(s-2) I\left(l_{1} \mid s\right)}{s}-\pi \beta\right)+O\left(\epsilon^{0}\right), \\
& j_{101101110}^{\text {cut21 }}=-\frac{1}{2} \pi I\left(l_{0} \mid s\right) \beta-\pi I\left(l_{2} \mid s\right) \beta+\pi(1+i \pi-\ln 2) \beta+\frac{\pi(s-2) I\left(l_{1}, l_{0} \mid s\right)}{2 s}+\frac{\pi(s-2) I\left(l_{1}, l_{2} \mid s\right)}{s} \\
& +\pi I\left(l_{0}, l_{1}, l_{0} \mid s\right)+2 \pi I\left(l_{0}, l_{1}, l_{2} \mid s\right)+\frac{\pi(s-1) I\left(l_{4}, l_{0}, l_{1} \mid s\right)}{2 s}-\frac{3 \pi(s-1) I\left(l_{4}, l_{1}, l_{0} \mid s\right)}{2 s} \\
& -\frac{3 \pi(s-1) I\left(l_{4}, l_{1}, l_{2} \mid s\right)}{s}+\frac{\pi I\left(l_{1} \mid s\right)(2 \ln 2 s-2 i \pi s+s-4 \ln 2+4 i \pi)}{2 s} \\
& +\frac{1}{2} \pi I\left(l_{0}, l_{1} \mid s\right)(-1-4 i \pi+4 \ln 2)+\frac{3 i \pi(s-1) I\left(l_{4}, l_{1} \mid s\right)(\pi+i \ln 2)}{s}+O\left(\epsilon^{1}\right), \\
& j_{101102110}^{\text {cut21 }}=\pi \beta-\frac{\pi(s-2) I\left(l_{1} \mid s\right)}{s}+O\left(\epsilon^{1}\right), \\
& j_{201101110}^{\mathrm{cut} 21}=\frac{3 i \pi(\pi+i \ln 2) I\left(l_{4}, l_{1} \mid s\right)}{s}+\frac{\pi I\left(l_{4}, l_{0}, l_{1} \mid s\right)}{2 s}-\frac{3 \pi I\left(l_{4}, l_{1}, l_{0} \mid s\right)}{2 s}-\frac{3 \pi I\left(l_{4}, l_{1}, l_{2} \mid s\right)}{s}+O\left(\epsilon^{1}\right), \\
& j_{101111100}^{\text {cut21 }}=\frac{1}{\epsilon}\left(\pi \beta-\frac{\pi(s-2) I\left(l_{1} \mid s\right)}{s}\right)+O\left(\epsilon^{0}\right), \\
& j_{110100111}^{\text {cut21 }}=\frac{1}{\epsilon}\left(\pi \beta-\frac{\pi(s-2) I\left(l_{1} \mid s\right)}{s}\right)+O\left(\epsilon^{0}\right), \\
& j_{120100111}^{\operatorname{cut} 21}=\frac{2 \pi I\left(l_{1}, l_{1}, l_{1} \mid s\right)}{s}-\frac{i \pi^{2} I\left(l_{1}, l_{1} \mid s\right)}{s}+O\left(\epsilon^{1}\right), \\
& j_{110101110}^{\text {cut } 21}=\frac{\pi(\beta s+i \pi s-4 i \pi)}{s}-\frac{\pi(s-2) I\left(l_{1} \mid s\right)}{s}-\frac{2 i \pi^{2} I\left(l_{1}, l_{1} \mid s\right)}{s}+\frac{4 \pi I\left(l_{1}, l_{1}, l_{1} \mid s\right)}{s}+O\left(\epsilon^{1}\right),
\end{aligned}
$$




$$
\begin{aligned}
& j_{110102110}^{\mathrm{cut} 21}=-\frac{i \pi^{2}(s-4) I\left(l_{0} \mid s\right)}{s}+\frac{\pi\left(i \pi \beta s^{2}-6 i \pi \beta s+2 s+4 i \pi \beta-8\right) I\left(l_{1} \mid s\right)}{(s-4) s}+\frac{\pi(s-4) I\left(l_{0}, l_{1} \mid s\right)}{s} \\
& -\frac{2 \pi\left(s^{2}-6 s+4\right) \beta I\left(l_{1}, l_{1} \mid s\right)}{(s-4) s}+\frac{\pi(s-4) I\left(l_{2}, l_{1} \mid s\right)}{s}-\frac{2 i \pi(-i \beta s+\pi \ln 2 s-4 \pi \ln 2-\pi)}{s}+O\left(\epsilon^{1}\right), \\
& j_{111101100}^{\text {cut21 }}=-\frac{\pi}{2} I\left(l_{0} \mid s\right) \beta-\pi I\left(l_{2} \mid s\right) \beta+\pi(1+i \pi-\ln 2) \beta+\frac{1}{2} \pi I\left(l_{0}, l_{1} \mid s\right)+\frac{\pi(s-2) I\left(l_{1}, l_{0} \mid s\right)}{2 s} \\
& -\frac{2 i \pi(2 \pi-i s \beta) I\left(l_{1}, l_{1} \mid s\right)}{s}+\frac{\pi(s-2) I\left(l_{1}, l_{2} \mid s\right)}{s}+\frac{8 \pi I\left(l_{1}, l_{1}, l_{1} \mid s\right)}{s}+\frac{\pi I\left(l_{4}, l_{0}, l_{1} \mid s\right)}{2 s}-\frac{3 \pi I\left(l_{4}, l_{1}, l_{0} \mid s\right)}{2 s} \\
& -\frac{3 \pi I\left(l_{4}, l_{1}, l_{2} \mid s\right)}{s}+\frac{\pi I\left(l_{1} \mid s\right)(2 i \pi \beta s+2 \ln 2 s-2 i \pi s+s-4 \ln 2+4 i \pi)}{2 s}+\frac{3 i \pi I\left(l_{4}, l_{1} \mid s\right)(\pi+i \ln 2)}{s}+O\left(\epsilon^{1}\right), \\
& +\frac{3 \pi I\left(l_{0}, l_{4}, l_{1}, l_{0} \mid s\right)}{2 s}+\frac{3 \pi I\left(l_{0}, l_{4}, l_{1}, l_{2} \mid s\right)}{s}+\frac{2 \pi I\left(l_{1}, l_{0}, l_{1}, l_{1} \mid s\right)}{s}-\frac{2 \pi I\left(l_{1}, l_{1}, l_{1}, l_{0} \mid s\right)}{s}-\frac{4 \pi I\left(l_{1}, l_{1}, l_{1}, l_{2} \mid s\right)}{s} \\
& +\frac{4 \pi I\left(l_{1}, l_{2}, l_{1}, l_{1} \mid s\right)}{s}+\frac{3 \pi I\left(l_{0}, l_{4}, l_{1} \mid s\right)(-i \pi+\ln 2)}{s}+\frac{4 i \pi I\left(l_{1}, l_{1}, l_{1} \mid s\right)(\pi+i \ln 2)}{s}+O\left(\epsilon^{1}\right), \\
& j_{121111100}^{\text {cut21 }}=\frac{\pi I\left(l_{1} \mid s\right)}{4 s \epsilon^{2}}+O\left(\frac{1}{\epsilon}\right) \\
& j_{11111110}^{\text {cut21 }}=\frac{1}{\epsilon^{2}}\left(\frac{i \pi^{2} \beta I\left(l_{1} \mid s\right)}{2(s-4) s}-\frac{\pi \beta I\left(l_{1}, l_{1} \mid s\right)}{(s-4) s}\right)+O\left(\frac{1}{\epsilon}\right), \\
& j_{121111110}^{\operatorname{cut} 21}=\frac{1}{\epsilon^{2}}\left(\frac{i \pi(i s \beta+2 \pi)}{4(s-4) s^{2}}+\frac{\pi(-i \pi \beta s-2 s+2 i \pi \beta+8) I\left(l_{1} \mid s\right)}{2(s-4)^{2} s^{2}}+\frac{\pi(s-2) \beta I\left(l_{1}, l_{1} \mid s\right)}{(s-4)^{2} s^{2}}\right)+O\left(\frac{1}{\epsilon}\right)
\end{aligned}
$$

\section{$(2,2)$ cuts}

$$
\begin{aligned}
& j_{101100010}^{\text {cut22 }}=\frac{1}{12} \pi\left(s^{2}+20 s+12\right) \beta-\frac{\pi(s-1)(s+2) I\left(l_{1} \mid s\right)}{s}+2 \pi I\left(l_{0}, l_{1} \mid s\right)+O\left(\epsilon^{1}\right), \\
& j_{201100010}^{\mathrm{cut} 22}=-\frac{1}{4} \pi(5 s+6) \beta+\frac{\pi\left(s^{2}+4 s-6\right) I\left(l_{1} \mid s\right)}{2 s}-2 \pi I\left(l_{0}, l_{1} \mid s\right)+O\left(\epsilon^{1}\right), \\
& j_{102100010}^{\text {cut } 22}=\frac{1}{\epsilon}\left(\frac{2 \pi(s-1) I\left(l_{1} \mid s\right)}{s}-\frac{1}{2} \pi(s+2) \beta\right)+O\left(\epsilon^{0}\right), \\
& j_{101100011}^{\text {cut22 }}=-\frac{1}{4} \pi(s-6) \beta+2 \pi I\left(l_{1}, l_{1} \mid s\right) \beta-\frac{\pi(s-3) I\left(l_{1} \mid s\right)}{s}-\pi I\left(l_{0}, l_{1} \mid s\right)+O\left(\epsilon^{1}\right), \\
& j_{101101110}^{\text {cut22 }}=-2 \pi I\left(l_{1}, l_{1} \mid s\right) \beta-\pi \beta+\frac{\pi(s-2) I\left(l_{1} \mid s\right)}{s}+\pi I\left(l_{0}, l_{1} \mid s\right)-\pi I\left(l_{0}, l_{0}, l_{1} \mid s\right) \\
& +\frac{2 \pi(s-2) I\left(l_{1}, l_{1}, l_{1} \mid s\right)}{s}+O\left(\epsilon^{1}\right), \\
& j_{101102110}^{\text {cut22 }}=\frac{1}{\epsilon}\left(\frac{\pi(s-2) I\left(l_{1} \mid s\right)}{2 s}-\frac{\pi \beta}{2}\right)+O\left(\epsilon^{0}\right), \\
& j_{201101110}^{\text {cut22 }}=\frac{4 \pi I\left(l_{1}, l_{1}, l_{1} \mid s\right)}{s}+O\left(\epsilon^{1}\right), \\
& j_{111100011}^{\mathrm{cut} 22}=-\pi \beta+\frac{\pi(s-2) I\left(l_{1} \mid s\right)}{s}-\frac{4 \pi I\left(l_{1}, l_{1}, l_{1} \mid s\right)}{s}+O\left(\epsilon^{1}\right), \\
& j_{111111110}^{\text {cut22 }}=\frac{2 \pi \beta I\left(l_{1}, l_{1} \mid s\right)}{(s-4) s \epsilon^{2}}+O\left(\frac{1}{\epsilon}\right), \\
& j_{121111110}^{\mathrm{cut} 22}=\frac{1}{\epsilon^{2}}\left(-\frac{2 \pi(s-2) I\left(l_{1}, l_{1} \mid s\right) \beta}{(s-4)^{2} s^{2}}+\frac{\pi \beta}{2(s-4) s}+\frac{2 \pi I\left(l_{1} \mid s\right)}{(s-4) s^{2}}\right)+O\left(\frac{1}{\epsilon}\right), \\
& j_{011111100}^{\mathrm{cut} 22 \mathrm{a}}=\left(-2 \pi I\left(l_{1}, l_{1} \mid s\right) \beta-\pi \beta+\frac{2 \pi I\left(l_{1} \mid s\right)}{s}+\frac{\pi(s-2) I\left(l_{0}, l_{1} \mid s\right)}{s}+\frac{\pi(s-4) I\left(l_{2}, l_{1} \mid s\right)}{s}\right)+O\left(\epsilon^{1}\right) \text {, } \\
& j_{011111110}^{\mathrm{cut} 22 \mathrm{a}}=\left(\frac{2 \pi I\left(l_{0}, l_{1}, l_{1}, l_{1} \mid s\right)}{s}-\frac{\pi I\left(l_{1}, l_{1}, l_{0}, l_{1} \mid s\right)}{s}-\frac{\pi I\left(l_{1}, l_{1}, l_{2}, l_{1} \mid s\right)}{s}\right)+O\left(\epsilon^{1}\right), \\
& j_{021111110}^{\mathrm{cut} 22 \mathrm{a}}=-\frac{\pi \beta I\left(l_{1}, l_{1} \mid s\right)}{(s-4) s \epsilon}+O\left(\epsilon^{0}\right), \\
& j_{111111110}^{\text {cut22a }}=\frac{\pi \beta I\left(l_{1}, l_{1} \mid s\right)}{(s-4) s \epsilon^{2}}+O\left(\frac{1}{\epsilon}\right), \\
& j_{211111110}^{\mathrm{cut} 22 \mathrm{a}}=\frac{1}{\epsilon^{2}}\left(\frac{\pi I\left(l_{1}, l_{1} \mid s\right) \beta}{(s-4) s^{2}}-\frac{\pi \beta}{2(s-4) s}+\frac{\pi I\left(l_{1} \mid s\right)}{2 s^{2}}\right)+O\left(\frac{1}{\epsilon}\right)
\end{aligned}
$$




\section{$(4,0)$ cuts}

$$
\begin{aligned}
& j_{100001110}^{\text {cutt40 }}=\frac{\pi s\left(s^{2}+82 s-128\right) f(s)}{24(s-16)^{2}}+\frac{\pi\left(s^{4}-22 s^{3}-1152 s^{2}+2432 s+4096\right) f^{\prime}(s)}{288(s-16)} \\
& +\frac{1}{288} \pi(s-4) s\left(s^{2}+40 s+64\right) f^{\prime \prime}(s)+O\left(\epsilon^{1}\right), \\
& j_{200001110}^{\text {cut } 40}=-\frac{9 \pi f(s) s^{2}}{8(s-16)^{2}}-\frac{1}{96} \pi(s-4)(5 s+16) f^{\prime \prime}(s) s \\
& -\frac{\pi(s+2)\left(3 s^{2}-176 s+512\right) f^{\prime}(s)}{96(s-16)}+O\left(\epsilon^{1}\right), \\
& j_{300001110}^{\text {cut } 40}=\frac{\pi\left(s^{2}+18 s-64\right) f(s)}{8(s-16)^{2}}+\frac{\pi(s+2)\left(s^{2}-44 s+128\right) f^{\prime}(s)}{96(s-16)} \\
& +\frac{1}{96} \pi(s-4) s(s+4) f^{\prime \prime}(s)+O\left(\epsilon^{1}\right), \\
& j_{100001111}^{\text {cut }}=-\frac{1}{96} \pi\left(s^{2}-26 s+64\right) f^{\prime}(s)-\frac{1}{96} \pi(s-16)(s-4) s f^{\prime \prime}(s)+O\left(\epsilon^{1}\right) \text {, } \\
& j_{101011110}^{\text {cut40 }}=-\frac{\pi I\left(r_{2} \mid s\right)}{6 s}-\frac{1}{24} \pi \beta I\left(\tilde{r}_{3} \mid s\right)+\frac{\pi I\left(l_{1}, \tilde{r}_{3} \mid s\right)}{12 s}-\frac{\pi(5 s-32) f(s)}{4(s-16)^{2}}+\frac{\pi(11 s-32) f^{\prime}(s)}{12(s-16)} \\
& -\frac{1}{24} \pi(s-4) s f^{\prime \prime}(s)+O\left(\epsilon^{1}\right), \\
& j_{102011110}^{\text {cut } 40}=-\frac{1}{48} \pi \beta I\left(\tilde{r}_{3} \mid s\right)+\frac{\pi(5 s+64) f(s)}{8(s-16)^{2}}+\frac{\pi\left(3 s^{2}-122 s+320\right) f^{\prime}(s)}{48(s-16)} \\
& +\frac{1}{16} \pi(s-4) s f^{\prime \prime}(s)+O\left(\epsilon^{1}\right), \\
& j_{101101110}^{\text {cutt40 }}=-\frac{1}{24} \pi I\left(r_{0} \mid s\right)+\frac{\pi(s-2) I\left(r_{2} \mid s\right)}{12 s}+\frac{1}{24} \pi \beta I\left(\tilde{r}_{3} \mid s\right)-\frac{\pi(s-2) I\left(l_{1}, \tilde{r}_{3} \mid s\right)}{24 s}-\frac{\pi\left(s^{2}-2 s+64\right) f(s)}{24(s-16)^{2}} \\
& +\frac{\pi(11 s-32) f^{\prime}(s)}{12(s-16)}-\frac{1}{24} \pi(s-4) s f^{\prime \prime}(s)+O\left(\epsilon^{1}\right), \\
& j_{201101110}^{\text {cut }}=\frac{\pi I\left(r_{2} \mid s\right)}{6 s}-\frac{\pi I\left(l_{1}, \tilde{r}_{3} \mid s\right)}{12 s}+O\left(\epsilon^{1}\right), \\
& j_{101102110}^{\text {cut } 40}=-\frac{1}{24} \pi \beta I\left(\tilde{r}_{3} \mid s\right)-\frac{\pi(5 s-32) f(s)}{4(s-16)^{2}}+\frac{\pi(11 s-32) f^{\prime}(s)}{12(s-16)}-\frac{1}{24} \pi(s-4) s f^{\prime \prime}(s)+O\left(\epsilon^{1}\right) \text {, } \\
& j_{110001111}^{\text {cut40 }}=-\frac{\pi I\left(r_{2} \mid s\right)}{12 s}+\frac{1}{48} \pi \beta I\left(\tilde{r}_{3} \mid s\right)+\frac{\pi I\left(l_{1}, \tilde{r}_{3} \mid s\right)}{24 s}-\frac{\pi(s+8) f(s)}{2(s-16)^{2}}-\frac{\pi\left(s^{2}-42 s+128\right) f^{\prime}(s)}{24(s-16)} \\
& -\frac{1}{24} \pi(s-4) s f^{\prime \prime}(s)+O\left(\epsilon^{1}\right), \\
& j_{120001111}^{\text {cut40 }}=\frac{\pi(3 s-8) \beta I\left(\tilde{r}_{3} \mid s\right)}{48(s-4) s}+\frac{\pi(5 s-8) f(s)}{6(s-16)(s-4) s}-\frac{1}{24} \pi f^{\prime}(s)+O\left(\epsilon^{1}\right) \text {, } \\
& j_{11111110}^{\text {cut40 }}=-\frac{\pi \beta I\left(l_{1}, r_{0} \mid s\right)}{24(s-4) s}+\frac{\pi \beta I\left(l_{1}, r_{2} \mid s\right)}{6(s-4) s}-\frac{\pi \beta I\left(l_{1}, l_{1}, \tilde{r}_{3} \mid s\right)}{12(s-4) s}+O\left(\epsilon^{1}\right), \\
& j_{121111110}^{\text {cut40 }}=\left(-\frac{\pi I\left(r_{0} \mid s\right)}{24(s-4) s^{2}}+\frac{\pi \beta I\left(r_{1} \mid s\right)}{8(s-4)^{2} s}+\frac{\pi I\left(r_{2} \mid s\right)}{6(s-4) s^{2}}-\frac{\pi(s-16) \beta I\left(\tilde{r}_{3} \mid s\right)}{96(s-4) s^{2}}-\frac{\pi \beta I\left(l_{0}, \tilde{r}_{3} \mid s\right)}{8(s-4)^{2} s}+\frac{\pi(s-2) \beta I\left(l_{1}, r_{0} \mid s\right)}{24(s-4)^{2} s^{2}}\right. \\
& \left.-\frac{\pi(s-2) \beta I\left(l_{1}, r_{2} \mid s\right)}{6(s-4)^{2} s^{2}}-\frac{\pi I\left(l_{1}, \tilde{r}_{3} \mid s\right)}{12(s-4) s^{2}}+\frac{\pi(s-2) \beta I\left(l_{1}, l_{1}, \tilde{r}_{3} \mid s\right)}{12(s-4)^{2} s^{2}}\right)+O\left(\epsilon^{1}\right)
\end{aligned}
$$


Open Access. This article is distributed under the terms of the Creative Commons Attribution License (CC-BY 4.0), which permits any use, distribution and reproduction in any medium, provided the original author(s) and source are credited.

\section{References}

[1] V.B. Berestetskii, E.M. Lifshitz, and L.P. Pitaevskii, Quantum Electrodynamics: Volume 4, Butterworth-Heinemann (1982).

[2] G. Källén and A. Sabry, Fourth order vacuum polarization, Kong. Dan. Vid. Sel. Mat. Fys. Med. 29 (1955) 1 [INSPIRE].

[3] P.A. Baikov and D.J. Broadhurst, Three loop QED vacuum polarization and the four loop muon anomalous magnetic moment, in 4th International Workshop on Software Engineering and Artificial Intelligence for High-energy and Nuclear Physics, (1995) [hep-ph/9504398] [INSPIRE].

[4] T. Kinoshita and M. Nio, Sixth order vacuum polarization contribution to the Lamb shift of the muonic hydrogen, Phys. Rev. Lett. 82 (1999) 3240 [Erratum ibid. 103 (2009) 079901] [hep-ph/9812442] [INSPIRE].

[5] T. Kinoshita and M. Nio, Accuracy of calculations involving $\alpha^{3}$ vacuum polarization diagrams: Muonic hydrogen Lamb shift and muon g-2, Phys. Rev. D 60 (1999) 053008 [hep-ph/9812443] [INSPIRE].

[6] T. Kinoshita and W.B. Lindquist, Parametric Formula for the Sixth Order Vacuum Polarization Contribution in Quantum Electrodynamics, Phys. Rev. D 27 (1983) 853 [INSPIRE].

[7] R.N. Lee and A.I. Onishchenko, $\epsilon$-regular basis for non-polylogarithmic multiloop integrals and total cross section of the process $e^{+} e^{-} \rightarrow 2(Q \bar{Q})$, JHEP $12(2019) 084$ [arXiv: 1909.07710] [INSPIRE].

[8] J.M. Henn, Multiloop integrals in dimensional regularization made simple, Phys. Rev. Lett. 110 (2013) 251601 [arXiv:1304.1806] [INSPIRE].

[9] R.N. Lee, Reducing differential equations for multiloop master integrals, JHEP 04 (2015) 108 [arXiv: 1411.0911] [INSPIRE].

[10] R.N. Lee, Libra: a package for transformation of differential systems for multiloop integrals, arXiv:2012.00279 [INSPIRE].

[11] R.N. Lee, LiteRed 1.4: a powerful tool for reduction of multiloop integrals, J. Phys. Conf. Ser. 523 (2014) 012059 [arXiv: 1310.1145] [INSPIRE].

[12] R.N. Lee LiteRed2: a major update of LiteRed package for IBP reduction of multiloop integrals, to be published.

[13] M. Beneke and V.A. Smirnov, Asymptotic expansion of Feynman integrals near threshold, Nucl. Phys. B 522 (1998) 321 [hep-ph/9711391] [INSPIRE].

[14] H. Cheng and T.T. Wu, Expanding protons: scattering at high energies, MIT, Cambridge, MA (1987) [INSPIRE].

[15] R. Bonciani, P. Mastrolia and E. Remiddi, Vertex diagrams for the QED form-factors at the two loop level, Nucl. Phys. B 661 (2003) 289 [Erratum ibid. 702 (2004) 359] [hep-ph/0301170] [INSPIRE]. 
[16] D. Maître, HPL, a mathematica implementation of the harmonic polylogarithms, Comput. Phys. Commun. 174 (2006) 222 [hep-ph/0507152] [INSPIRE].

[17] D. Maître, Extension of HPL to complex arguments, Comput. Phys. Commun. 183 (2012) 846 [hep-ph/0703052] [INSPIRE].

[18] H. Ferguson and D. Bailey, A polynomial time, numerically stable integer relation algorithm, RNR-91-032 (1992)

[https://www.nas.nasa.gov/assets/pdf/techreports/1991/rnr-91-032.pdf].

[19] A.V. Kotikov, Differential equations method: New technique for massive Feynman diagrams calculation, Phys. Lett. B 254 (1991) 158 [INSPIRE].

[20] A.V. Kotikov, New method of massive Feynman diagrams calculation, Mod. Phys. Lett. A 6 (1991) 677 [INSPIRE].

[21] A.V. Kotikov, Differential equations method: The Calculation of vertex type Feynman diagrams, Phys. Lett. B 259 (1991) 314 [InSPIRE].

[22] A.V. Kotikov, Differential equation method: The Calculation of $N$ point Feynman diagrams, Phys. Lett. B 267 (1991) 123 [Erratum ibid. 295 (1992) 409] [INSPIRE].

[23] E. Remiddi, Differential equations for Feynman graph amplitudes, Nuovo Cim. A 110 (1997) 1435 [hep-th/9711188] [INSPIRE].

[24] T. Binoth and G. Heinrich, An automatized algorithm to compute infrared divergent multiloop integrals, Nucl. Phys. B 585 (2000) 741 [hep-ph/0004013] [INSPIRE].

[25] T. Binoth and G. Heinrich, Numerical evaluation of multiloop integrals by sector decomposition, Nucl. Phys. B 680 (2004) 375 [hep-ph/0305234] [INSPIRE].

[26] T. Binoth and G. Heinrich, Numerical evaluation of phase space integrals by sector decomposition, Nucl. Phys. B 693 (2004) 134 [hep-ph/0402265] [INSPIRE].

[27] G. Heinrich, Sector Decomposition, Int. J. Mod. Phys. A 23 (2008) 1457 [arXiv:0803.4177] [INSPIRE].

[28] C. Bogner and S. Weinzierl, Resolution of singularities for multi-loop integrals, Comput. Phys. Commun. 178 (2008) 596 [arXiv:0709.4092] [INSPIRE].

[29] C. Bogner and S. Weinzierl, Blowing up Feynman integrals, Nucl. Phys. B Proc. Suppl. 183 (2008) 256 [arXiv: 0806.4307] [INSPIRE].

[30] T. Kaneko and T. Ueda, A Geometric method of sector decomposition, Comput. Phys. Commun. 181 (2010) 1352 [arXiv:0908. 2897] [INSPIRE].

[31] A.V. Smirnov, FIESTA4: Optimized Feynman integral calculations with GPU support, Comput. Phys. Commun. 204 (2016) 189 [arXiv:1511.03614] [INSPIRE].

[32] O.V. Tarasov, Connection between Feynman integrals having different values of the space-time dimension, Phys. Rev. D 54 (1996) 6479 [hep-th/9606018] [InSPIRE]. 